$\mathbb{T}$ periodica polytechnica

Civil Engineering

$56 / 1(2012) 97,106$

doi: 10.3311/pp.ci.2012-1.11

web: http://www.pp.bme.hu/ci

(c) Periodica Polytechnica 2012

RESEARCH ARTICLE

\section{Detecting the chaotic nature of advection in complex river flows}

\author{
Márton Zsugyel / K. Gábor Szabó / Zs. Melinda Kiss / János Józsa / Giuseppe \\ Ciraolo / Carmelo Nasello / Enrico Napoli / Tamás Tél
}

Received 2011-03-03, revised 2011-06-23, accepted 2011-10-18

\begin{abstract}
In order to detect signatures of chaotic advection in river surface motion, surface buoys equipped with GPS were deployed in a field experiment in River Danube, Hungary. The buoys were released in the vicinity of groynes where complex mixing processes occur. A detailed analysis of the trajectories was carried out, focusing on the time evolution of the distance between buoy pairs. The analysis included the determination and comparison of local Lyapunov exponents and prediction times of finite-time hyperbolic behaviour, which is related to strong mixing. Despite of the small number of applied buoys we found evidence on Lagrangian chaos in the wake of a groyne field. In order to supplement the field data obtained by this, inherently Lagrangian, approach, experiments in a small-scale laboratory model were also carried out, in which the Lagrangian surface dynamics was detected by following the motion of numerous floaters using particle tracking velocimetry (PTV).
\end{abstract}

\section{Keywords}

River hydraulics · groyne (groin) · chaotic mixing · Lagrangian transport $\cdot$ Particle Tracking Velocimetry $($ PTV)

\section{Márton Zsugye}

\section{K. Gábor Szabó}

Budapest University of Technology and Economics, Department of Hydraulic and Water Resources Engineering, H-1111 Budapest, Múegyetem rakpart 3. Kmf. 12, Hungary

\section{Zs. Melinda Kiss}

János Józsa

Budapest University of Technology and Economics, Department of Hydraulic and Water Resources Engineering, H-1111 Budapest, Múegyetem rakpart 3. Kmf. 12, Hungary

\section{Giuseppe Ciraolo \\ Carmelo Nasello \\ Enrico Napoli}

University of Palermo, Department of Civil, Environmental and Aerospace Engineering, I-90128 Palermo, Viale delle Scienze - Ed. 8, Italy

Tamás Tél

Eötvös University, Institute for Theoretical Physics, H-1117 Budapest, Pázmány Péter sétány $1 / \mathrm{A}$, Hungary

\section{Introduction}

Mixing properties in fluvial conditions have long been investigated as important environmental features. The areas in between or downstream of river groynes are extremely challenging river segments due to their complex and ever varying flow conditions that influences sediment motion, bed formation, transport of nutrients and pollutants. When dealing with pollutant plumes in rivers, it has often been experienced that the application of a naive Fickian numerical approach results in poor matching to the field data. It is particularly true in the vicinity of groyne fields where the spatial complexity of the flow field coupled with an inherent unsteadiness, larger in scale than the one of the typical turbulent eddies, can result in very complicated, occasionally distorted spreading. The misinterpretation of the mixing mechanism in such zones can have then a significant impact on the overall accuracy of the calculations. Field data obtained by traditional, Eulerian, methods have shown their limited applicability especially at reaches mentioned above. In fact, a more sound knowledge of the hydrodynamic conditions, more exactly, information on how water particles move both individually and relative to each other - a Lagrangian concept - would substantially improve our ability to predict plume evolutions.

In fact, in complex velocity fields with inherent timedependence the basic mechanism of mixing is often chaotic advection, which is best handled as Lagrangian transport (Aref, 1984). Structures like hyperbolic and elliptic points, stable and unstable manifolds have been used for a long time to describe the evolution of trajectories in abstract dynamical systems. By applying the methods of chaos theory in the context of fluid dynamics, it is possible to identify spatial structures that govern the flow and to locate areas where the most effective spreading occurs. Such fluid dynamical structures include vortex boundaries, barriers and channels of transport, or lines of strong stretching coupled with contraction.

The most characteristic feature of chaotic motion is its (exponential) sensitivity to initial conditions. The traditional way to characterize this feature is the determination of the standard Lyapunov exponent of chaotic advection (see e.g. Tél and Gruiz, 2006). In a fluid dynamical application, chaotic motion man- 
ifests itself in an exponential growth of the distance between nearby fluid particles; if the initial distance $\Delta r_{0}$ is small, the growth of the distance in time $t$ is proportional to an exponential factor:

$$
\Delta r(t)=\Delta r_{0} \cdot e^{\lambda t}
$$

This rule implies a very rapid increase resulting in strong spreading of particles and stretching of pollutant patches. The growth rate $\lambda$ is called the local Lyapunov exponent, which can serve as a quantitative measure of the strength of particle dispersion to characterise mixing. The reciprocal of the Lyapunov exponent can be interpreted as the local prediction time: the characteristic time scale under which information on the initial conditions is being lost in a system. After this time, the prediction of the fluid particle positions is not possible with traditional tools.

Non-chaotic locations in the flow field, where the dispersion of nearby particles is slower than exponential (like in case of Fickian diffusion), indicate poorer mixing (that is lower dilution). The extreme case, in which particles stay close to each other all along their advection history, corresponds to particle trapping. Typically, long-time particle trapping occurs in the cores of vortices.

In chaotic motion, trajectories of initially close particles separate along the stretching directions: such a behaviour is characteristic in the vicinity of the reattachment (stagnation) point of recirculation at groyne fields. Note that if the flow field were in steady state, the fluid would be motionless at such points. However, in cases of even slight unsteadiness these stagnation points, as Eulerian features, are only instantaneous structural elements of the flow. In such unsteady conditions the Lagrangian equivalent hyperbolic or saddle points have to be used; these are actually fluid particles themselves that move with the fluid and at any instant of time stable and unstable manifolds belong to them.

A number of papers deal with mixing that occurs in conditions similar to the ones described above, but only few of them look at it from a Lagrangian point of view. An example of this is the work of Weitbrecht (2004) who investigated the fluvial mixing processes, though in laboratory conditions, at groyne fields with Lagrangian particle tracking method. However, his modelling goal was to establish the Lagrangian equivalent of conventional Fickian diffusion, in which he tracked particles individually and did not pay attention to inter-particle distance evolution. In fact, tracking the distance of pairs of particles starting close to each other initially provides a good insight to the mixing process.

Most of the papers use the conventional Eulerian approach when studying groyne flow fields. Mazijk (2002) used the Rhine Alarm-Model to investigate the influence of groyne fields on the transport of a spill. He found that a lag coefficient describe the influence of the groyne field, however in low water conditions this coefficient is not able to characterise well the mixing properties. Engelhardt and his group (2004) investigated another aspect of the groyne fields: the spatial distribution of phytoplank- ton in river environment. They found that the spatial patterns of a number of phytoplankton characteristics were influenced more by local flow characteristics than by other biological processes. The aspect ratio (the ratio of groyne length to the distance of the groynes) was found to be an important parameter in determining the pattern of phytoplankton. Nevertheless, they underlined the importance of the further investigation on mixing processes even by numerical models and field studies. Many papers focus on laboratory model measurements of exchange processes in groyne fields (e.g. Uijttewaal et al., 2001; Weitbrecht et al., 2002; or Uijttewaal, 2005). One of the first works in this context was published by Uijttewaal et al. in 2001. They investigated experimentally the impact of several geometrical and hydraulic conditions of the groyne field on the mixing processes and the velocity field. They found two different types exchange. The first one is in the mixing layer of the large eddies generated by the groyne; the second is due the distortion of the larger eddies shed from an upstream groyne. They used dye concentration measurements to quantify the mixing process and particle tracking velocimetry (PTV) to connect the results to the surface velocity field. Uijttewaal in 2005 made some further investigation in some different geometry and material of groynes. $\mathrm{He}$ experienced that the flow structure cannot be considered twodimensional (2D) but it is influenced locally with vertical effects when the groynes are submerged in the water. Hence, applying depth averaged numerical models or even three dimensional (3D) models with too coarse resolution does not give good results in these cases. Weitbrecht and his associates also investigated groyne fields in the laboratory using particle image velocimetry (PIV) technique together with dye concentration measurements. They determined e.g. the mass exchange coefficient (Kurzke et al. 2002) in various groyne field geometries. Mean surface velocity field in the dead zone, turbulent flow characteristics and vorticity field was also determined in a simple case (Weitbrecht et al., 2002). [An overview of the PTV and PIV techniques is given by e.g. Sokoray-Varga and Józsa (2008).]

In this paper, we present the first analysis and evaluation of our field investigations carried out on the River Danube in Hungary. In order to assist navigation, the Danube has undergone thorough river training. Stand-alone groynes and groyne sequences are typical structures along the river. In order to detect mixing features with occasional chaotic advection behaviour various numbers of buoys (at most 5) with on-board GPS receiver and data storage units were simultaneously released close to each other at several locations in the vicinity of groynes. A detailed analysis of their trajectories was then carried out: the measurement being inherently Lagrangian, we focused on the temporal evolution of the bouy-to-buoy distances.

The paper is organised as follows. In Section 2 we describe the main conditions of our field measurements including the site description, the applied buoys and also the data processing. In Section 3 we present the analysis of the recorded data. In Section 4 we describe the results of our laboratory PTV measure- 
ments. Finally, in Section 5 we draw conclusion and discuss the results.

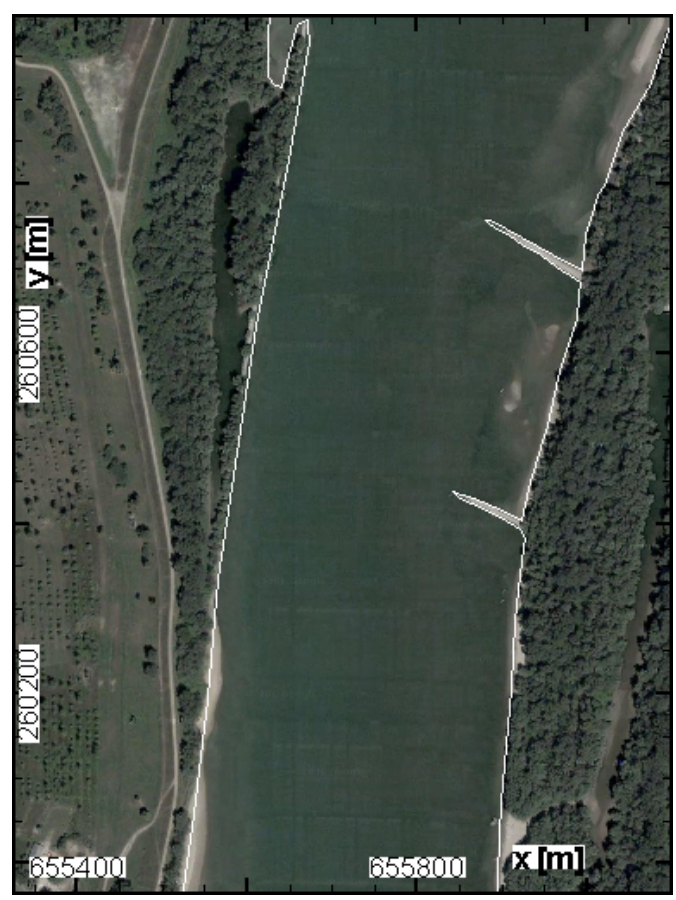

Fig. 1. The test reach on the River Danube near Göd, Hungary, (with flow from top to down) in low water conditions

\section{Field measurements on the Danube}

2.1 Measurement site, times and environmental conditions

The study area for buoy release was chosen in the River Danube near Göd, some $20 \mathrm{~km}$ upstream of Budapest, close to the hydrometric station of the Budapest University of Technology, providing a long term base for the investigations. In this area there are several groynes. The test reach we chose around the geographical position N47.688648, E19.125502, presented in Fig. 1. contains two groynes. The Danube flows from North to South in this stretch. The upstream and the downstream groynes are about 100 and 76 meter long, respectively, and the distance between them is about 300 meters. The first measurement campaign was carried out in the autumn of 2009 with three buoys in slowly rising, rather low water conditions and partly emergent groynes. The buoys were released upstream of the groyne tip, close to each other. Out of eight releases, six gave acceptable quality data. During the second campaign in the summer of 2010, the hydraulic situation was different: high and gradually decreasing water levels prevailed, the groynes were submerged so the buoys were able to cross over them. In this second campaign six buoys were used (however, because of telecommunication problems, at most five of them recorded useful data at the same time) and 12 out of the 14 releases provided valuable data. In Tab. 1 we summarized the major conditions of the two measurement campaigns.

\subsection{The floater buoys}

The experimental drifter buoys (shown in Fig. 2) we used were equipped with GPS receiver for positioning, data storage unit, GSM transceiver for data transfer and remote communication and batteries. The electronics were contained in the watertight housing. The approximate sizes of the buoys are $16 \mathrm{~cm}$ in diameter (on the top) and $22 \mathrm{~cm}$ in height. On the top of the buoy, the GPS antenna, a connector - for battery charging and direct cable communication - and a magnetic switch are located (Fig. 2).

The buoys have originally been developed for marine use. In order to adjust them to fluvial conditions, a pair of wings and a fixed rudder have been mounted on the buoys (also shown in Fig. 2). The wings are meant to increase the submerged surface area, assisting to obtain a drift velocity as close to the local mean flow velocity of the uppermost $2 \mathrm{dm}$ layer as possible. Each wing is $30 \mathrm{~cm}$ long and $12 \mathrm{~cm}$ high. The diameter of the wing appendage, approximately $8 \mathrm{dm}$, was sized to meet the theoretical accuracy of the GPS unit, which is less than a meter. The buoys have been designed and manufactured in cooperation between an Italian company and the Department of Hydraulic Engineering of the University of Palermo.

A small inflatable tube (not shown in the figure) has been attached to each buoy to counteract the reduced buoyancy due to the sweet water environment and to provide extra buoyancy for supporting the wing appendage.

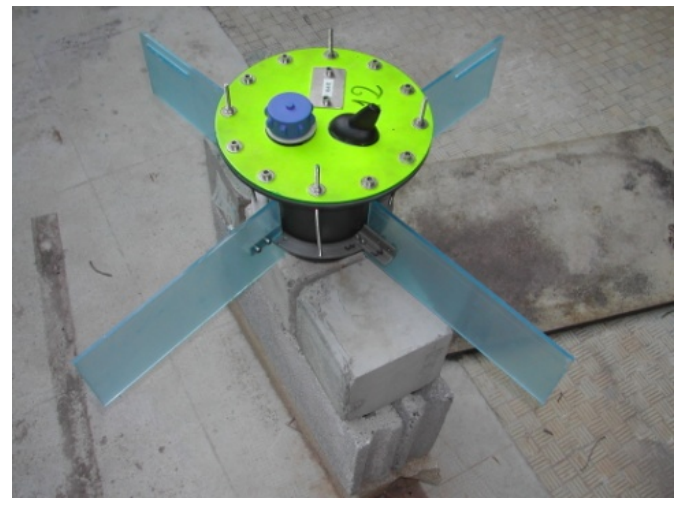

Fig. 2. Photograph of the experimental buoy

\subsection{The experimental releases}

At the beginning of each release the buoys were deployed from a motor boat, all at the same time, in such a manner to minimise their initial distance. We managed to keep the initial distances below $2 \mathrm{~m}$, in the magnitude of the GPS resolution. At the end of the release, the buoys were collected by guarding motor boats. The GPS units were set a priori to record position signals in every six seconds, which were stored in the memory and were downloaded a posteriori via GSM or direct cable connection. Unfortunately, since this technology cannot be entirely free from signal noise or miscommunication, occasionally we obtained gaps in the position time series and false positions. 
Tab. 1. Summary of the major measurement conditions during the field campaigns

\begin{tabular}{ccccccc}
\hline Campaign & Date & $\begin{array}{c}\text { Water } \\
\text { level }\end{array}$ & $\begin{array}{c}\text { Water level } \\
\text { tendency }\end{array}$ & $\begin{array}{c}\text { Number of } \\
\text { releases }\end{array}$ & $\begin{array}{c}\text { Successful } \\
\text { releases }\end{array}$ & $\begin{array}{c}\text { Released } \\
\text { buoys }\end{array}$ \\
\hline $1^{\text {st }}$ & $2009-11-04 / 05$ & low & $+2.4 \mathrm{dm} /$ day & 8 & 6 & 3 \\
\hline $2^{\text {nd }}$ & $2010-08-12 / 13$ & high & $-3.2 \mathrm{dm} /$ day & 14 & 12 & $4-5$ \\
\hline
\end{tabular}

\subsection{Data processing}

Processing the data started with segmenting the position time series of each buoy and combining them corresponding to individual releases. In cases when a buoy trajectory showed anomalous, erroneous behaviour, the data were discarded right away. Then linear interpolation was applied in between subsequent position data, mainly because individual buoys were not synchronised and, partly, to fill in small gaps of missing data. This preprocessing of data has no significant impact on the qualitative detection and characterisation of the chaotic behaviour. A sensitivity study of chaotic advection on the accuracy of the underlying flow field supported this fact (Pattantyús-ábrahám et al., 2008).

Then the following procedure was carried out for each release. First the buoy trajectories of the release were plotted (e.g. Figs. 3 and 4 a below). Then for each buoy pairs the separation distance between the buoys were plotted against time in a semi-logarithmic diagram (e.g. Figs. $3 \mathrm{~b}$ and $4 \mathrm{p}$ below). In this diagram we identified segments with constant slopes, which indicate exponential separation according to Eq. (1), as chaotic behaviour, and applied a standard linear regression (in the semilogarithmic representation) to find the corresponding local Lyapunov exponent, $\lambda$. Choosing a linear segment within a graph involves a significant degree of subjectivity. To lessen this subjective factor we had to lay down the following rules:

1 the determination coefficient $R^{2}$ of the linear regression had to be greater than 0.9 ;

2 the duration of the particular straight line segment had to exceed the prediction time, $1 / \lambda$;

3 the residuals of the linear regression should not have shown significant systematic trend (this involved, unfortunately, another subjective decision).

We admitted those segments in the further study that satisfied the three rules above. From the 19 (64) subjectively identified straight-line segments of the $1^{\text {st }}\left(2^{\text {nd }}\right)$ campaign only 10 (38) were admitted eventually, respectively, the others were excluded. We list the major data of the time intervals admitted with reliable Lyapunov exponents in Tab. 2,5 in the Appendix on a day-by-day basis. The admitted time intervals were marked in the diagrams and the release data so processed were passed to data analysis.

\section{Analysis of field data}

In this Section, first we present the results obtained for two selected releases, characteristic to the low and the high water conditions. Then we compare the spatial distributions of the local Lyapunov exponents found in different conditions.

\subsection{Low water conditions}

Fig. 3 a shows the trajectories of Release 8 of the $1^{\text {st }}$ campaign. The $x$ and $y$ axes correspond to the Easterly and Northerly horizontal coordinates in meters, the two gray straight line segment represent the locations of the two groynes, and different curves show the trajectories of the buoys. Three buoys were deployed in this release, which lasted 26 minutes. During this period, the initially small distances among the buoys - at most about 2 meters - grew to several hundred meters. Fig. 3 p shows the separation history of the all buoy pairs on a semi-logarithmic plot. In this plot, the admitted segments with constant slopes are highlighted indicating exponential separation. In the first minute every pair show an intensive separation that occurs within the highshear zone immediately downstream of the separation point at the groyne tip. The high local Lyapunov exponent $\lambda_{1}$ seems to be characteristic to this flow region. However, the separation between buoys \#06 and \#08 started about half minute later than between the buoy pairs containing buoy \#07. This small difference was enough to drive the buoys to completely different 'fate'. Two of the buoys, \#06 and \#08, were trapped in the recirculation zone of the upstream groyne. After that, they stayed relatively close to each other: their final separation was about 30 meters, however their greatest distance was about 55 meters. This latter size compares to the magnitude of the groyne length, indicating that the primary eddy in the separation zone of the groyne trapped the buoys. The third buoy - \#07, marked with dotted line - missed the trap of the recirculation zone and stayed in the main stream. This resulted in a large separation, nevertheless the buoy was eventually trapped by the second groyne. It is noteworthy that after buoys \#06 and \#08 had been caught in the recirculation zone, the exponential separation from \#07 was continuing for five more minutes, but with significantly lower local Lyapunov exponents, $\lambda_{2}$ and $\lambda_{3}$. During this period buoys \#06 and \#08 seem to be staying in a smaller (cca. 20 meter sized) eddy.

\subsection{High water conditions}

In the $2^{\text {nd }}$ campaign the hydraulic conditions were different (see Tab. 1) resulting in qualitatively different, much smoother buoy trajectories. As it is discussed by Uijttewaal (2005) in detail, the recirculation zone disappears when the groyne is submerged. Therefore, the main causes of the separation become the turbulent structures of the river. In Fig. $4 \mathrm{a}$ a typical release 


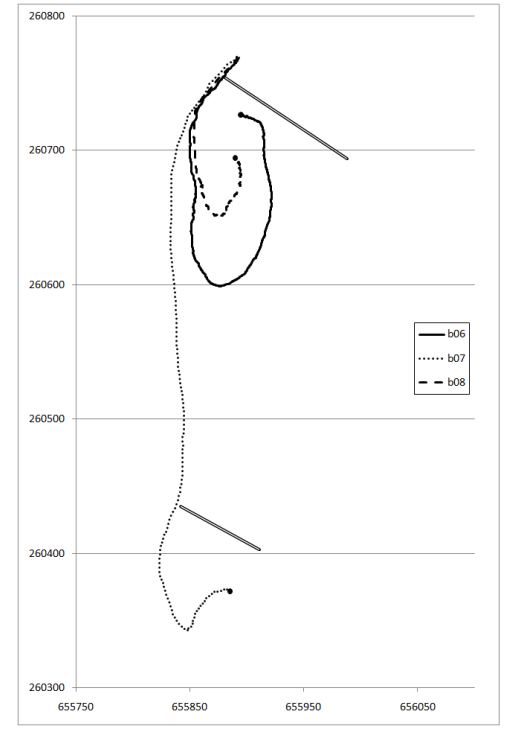

(a)

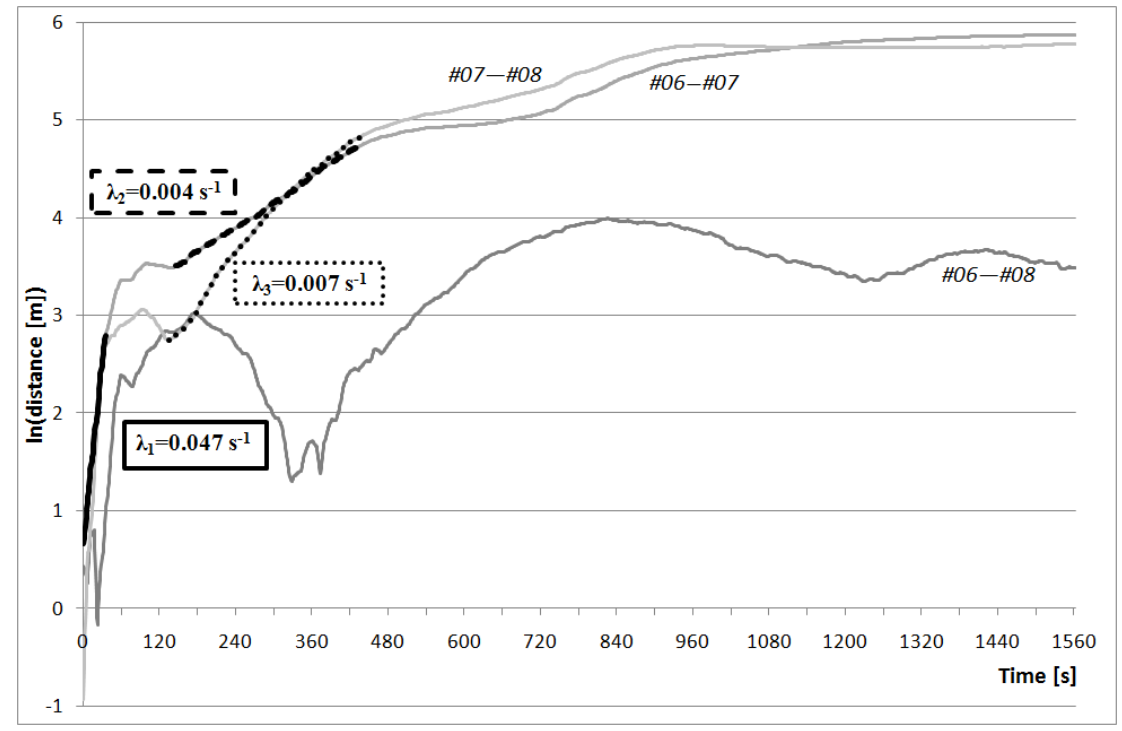

(b)

Fig. 3. Release 8 of the $1^{\text {st }}$ campaign, 2009-11-05. (a) Buoy trajectories. (b) Buoy pair distances vs. time in semi-logarithmic plot

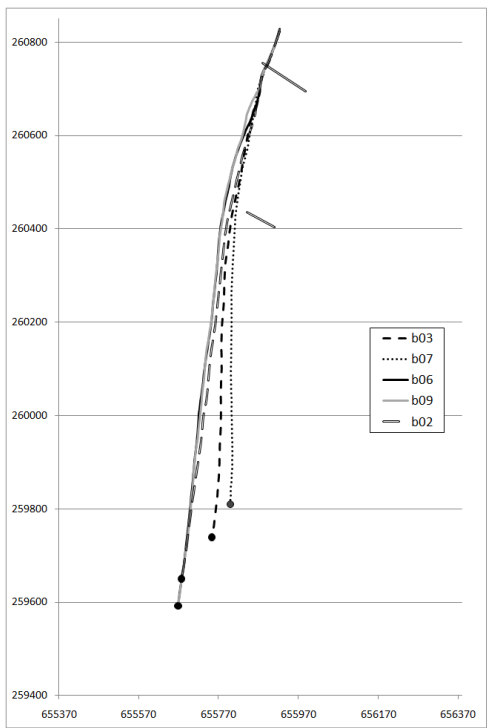

(a)

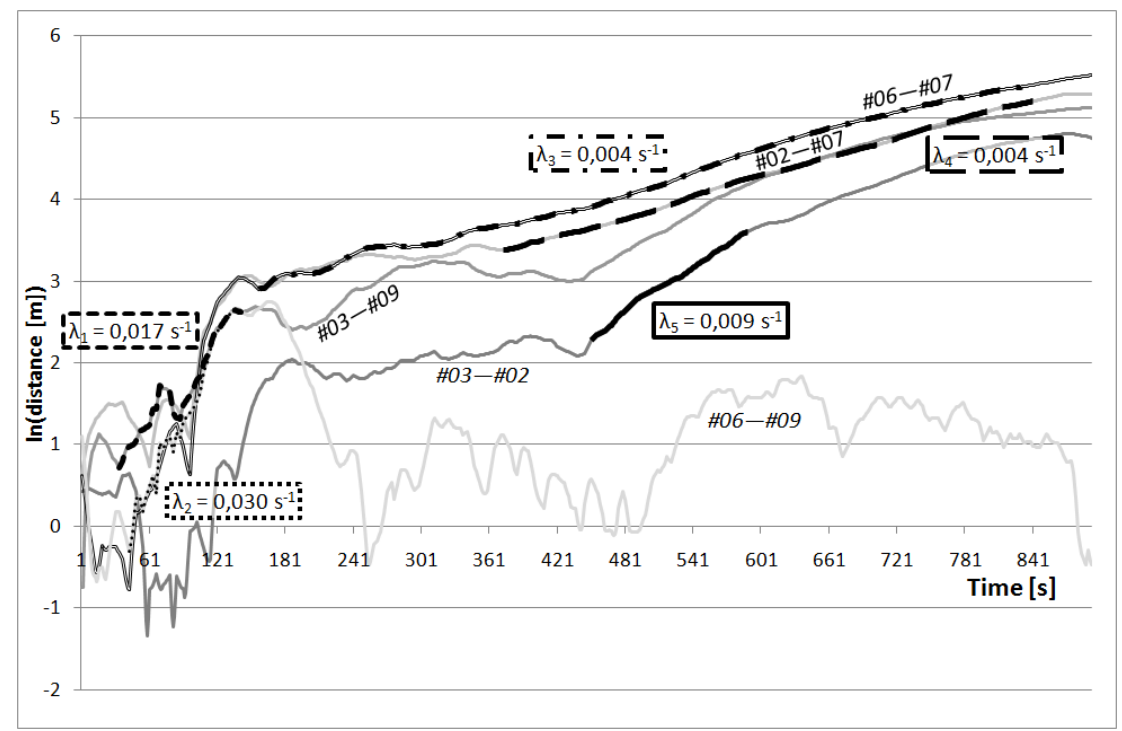

(b)

Fig. 4. Release 7 of the $2^{\text {nd }}$ campaign, 2010-08-12. (a) Buoy trajectories. (b) Buoy pair distances vs. time in semi-logarithmic plot 
from the $2^{\text {nd }}$ campaign is presented. This release involved five buoys and took 15 minutes. The largest distance between two buoys by the end of the release grew up to about 250 meters. Fig. 4 p shows the distance as a function of time of some particular buoy pairs. Although using five buoys would imply ten different lines to be plotted, for better insight we displayed only those that have different exponential segments. The buoys behaved differently from each other. The most peculiar history belongs to buoys \#06 and \#09. They underwent a 98 s long exponential departure while crossing over the upstream groyne's crest (marked with dotted line on Fig. $4 \mathrm{~b} ; \lambda_{2}=0.03 \mathrm{~s}^{-1}$ ), reaching a maximum distance of about 15 meters downstream of the groyne. Then this pair approached each other again and stayed close together, as if they were trapped in a small-scale persistent vortex drifting in the main stream of the river. Meanwhile the other buoys spread continuously. Actually, while crossing over the upstream groyne in the first two minutes, every pair showed a similar sudden increase in the distance. After passing the groyne, a long, continuous, but not too steep $\left(\lambda_{3}=0.004 \mathrm{~s}^{-1}\right)$ slope (marked with point-dash line) is observable between the fastest buoys (\#06 and \#09) and the slowest one (\#07). Most of the other curves show just a slight growth of distances during the time when the buoys were between the two groynes. The long and steady departure between buoys \#02 and \#07 (long dashed line, $\lambda_{4}$ ) started when the buoys passed by the downstream groyne. Some seconds later a more intensive separation started between buoys \#03 and \#02 (continuous black line, $\lambda_{5}$ ); even though they had been keeping approximately the same distance in between the two groynes, they were directed apart in the shear zone of the downstream groyne by a turbulent structure.

\subsection{Localization of the Lyapunov exponents}

It is interesting to see the spatial distribution of the trajectory sections over which the buoys exhibit exponential departure, indicating positive values of the local Lyapunov exponent. We present in Fig. 5 these segments. In the plots the different colour of the lines means that the exponent's value fall in a different interval: the separation is more significant the darker the line colour is.

In the Fig. 5a, the exponents of the first campaign are displayed. The identified segments are usually short, located in the vicinity of the groynes, and mostly high values are present. In the $2^{\text {nd }}$ campaign the general picture is very different due to the combined effect of the water level and the different flow structures formed by the groyne field. One of the main differences is that exponential stretching occurs not only in the vicinity of the groynes, but further downstream, close to the main stream, as well. The second difference appears in the duration and the strength of the segments: the exponents were relatively smaller, but lasted much longer in time than in the $1^{\text {st }}$ campaign.

Another important observation becomes apparent by comparing the results of the two measurement days of the $2^{\text {nd }}$ campaign in Figs. 5 5 and 5r. On the first day at higher water level, when the groynes were more submerged, the typical value of the Lyapunov exponents was $0.004 \mathrm{~s}^{-1}$, while on the next day, when the water level was about $30 \mathrm{~cm}$ lower, this characteristic value grew up to $0.01 \mathrm{~s}^{-1}$. It means that despite lower flow regimes in general imply lower discharge, thus lower overall velocity magnitude, near such complex river geometries as groynes they can even intensify space-time flow features and cause significant increase in Lagrangian spreading.

\section{Laboratory measurements using PTV technique}

The possibilities to obtain extensive data in actual field conditions are rather limited. The major factors include the number of available buoys and personnel, since continuous human watch is required to guard the buoys from river traffic and, especially in high water conditions, from collision with floating debris. Therefore, understanding the Lagrangian structures of fluvial surface motion requires the comparison of field data to other sources of information as well. On one hand, numerical experiments can be performed, this will be discussed in Section 5. On the other hand, small-scale laboratory experiments can also provide useful data. The above mentioned limitations can be easily overcome in the protected environment of a laboratory experiment. In what follows we present the experience obtained from measurements performed in the Hydraulic Laboratory of the Budapest Technical University.

The experiments were made in a straight, rectangular open test channel. The channel built for this purpose is $1 \mathrm{~m}$ wide and has a gross length of approximately $8 \mathrm{~m}$, in the middle of which the test section is located. The water level and the flow rate can be controlled independently, allowing the flexible variation of the flow conditions. In the case we present below the discharge rate was $4.52 \mathrm{dm}^{3} / \mathrm{s}$ and the water level was held at $7 \mathrm{~cm}$. During the experiments one or two groyne models were positioned in the test section in several configurations, varying their attitude angle with respect to the channel and their distance. For drifters $9 \mathrm{~mm}$ diameter white polyethylene "buoys" were deployed well upstream of the test section. In a typical "release" their number was above one thousand. The number of floater particles, the location and the way of their deployment was chosen to provide an optimum density of particles in the test section: high enough to have a satisfactory coverage of the most important flow features, but low enough to avoid particle-particle interactions. The motion of the floater trajectories was followed by a CCD camera, fixed above the test section. The camera provided gray-scale pictures of $1392 \times 1040$ resolution at $30 \mathrm{~Hz}$ frequency, which was subsequently processed by particle tracking algorithms. This PTV apparatus and the corresponding algorithms were developed by Sokoray-Varga and Józsa (2008), we refer their paper for the detailed description of the PTV system and for the discussion of applicability of the floater particles.

In Fig. 6 we present the particle tracks observed over a 60 frame (i.e. 2 s) long period downstream of a groyne. In order to capture events of particle exchange between the main stream 


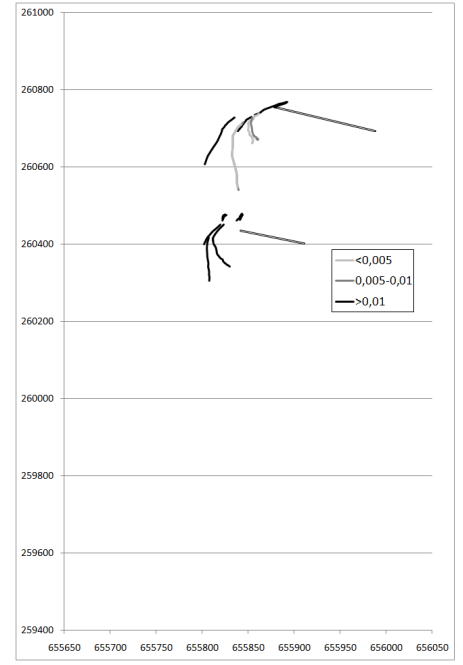

(a)

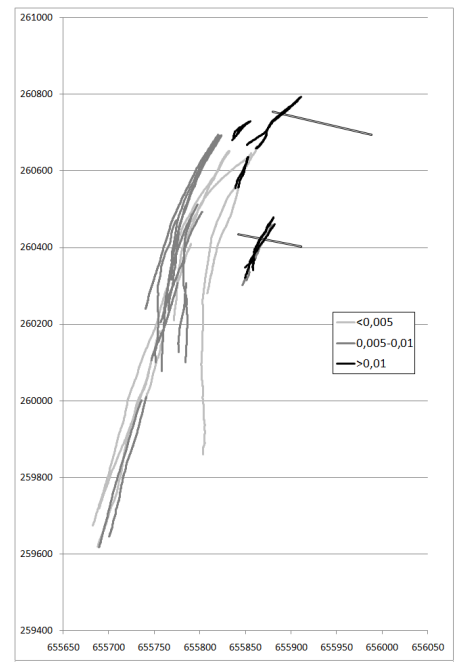

(b)

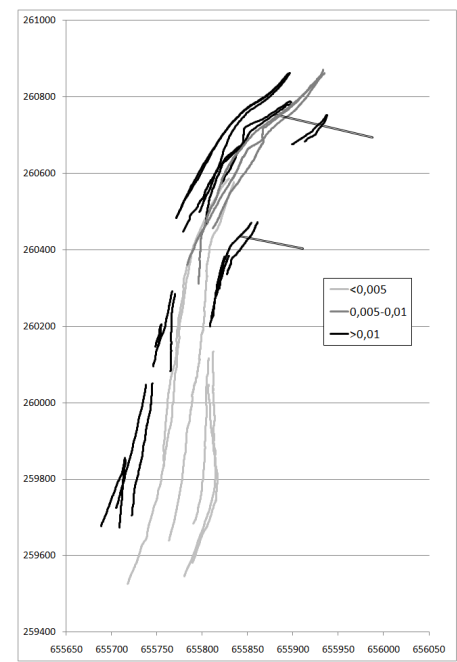

(c)

Fig. 5. Spatial distribution of admitted Lyapunov-exponents $\left[\mathrm{s}^{-1}\right]$ in the $1^{\text {st }}$ campaign (a); on $12^{\text {th }}$ (b) and $13^{\text {th }}$ (c) of August, 2010 in the $2^{\text {nd }}$ campaign

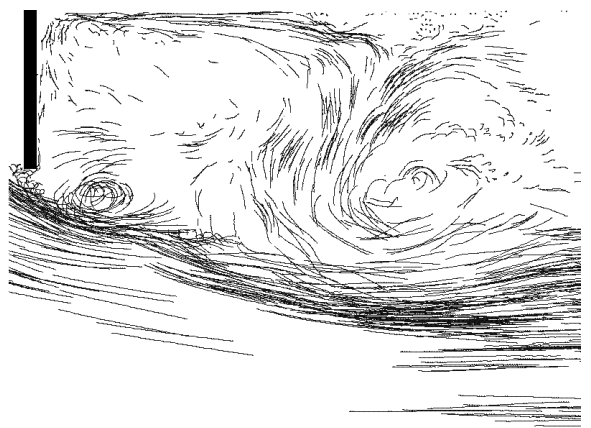

(a)

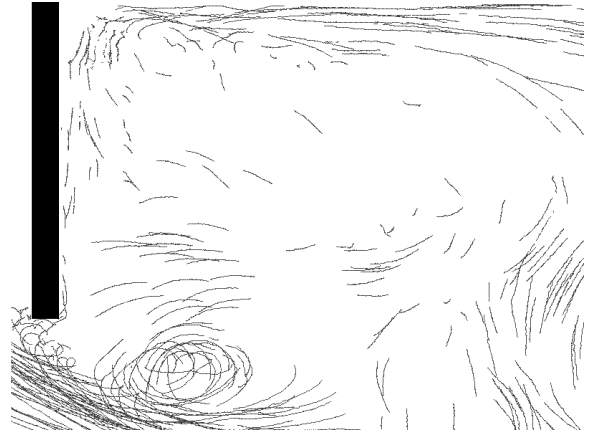

(b)

Fig. 6. (a) Particle tracks over a $2 \mathrm{~s}$ long period and (b) the enlargement of the recirculation zone downstream of the groyne (black rectangle).

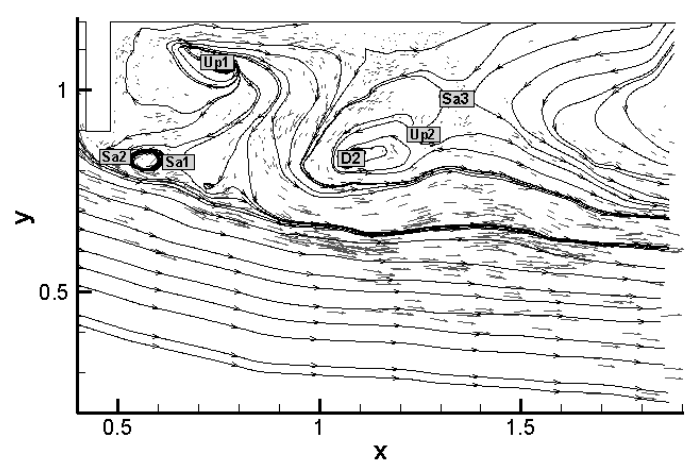

(a)

Fig. 7. The instantaneous streamlines with the positions of some major flow structures at the beginning (a) and the end (b) of the period shown in Fig. 6

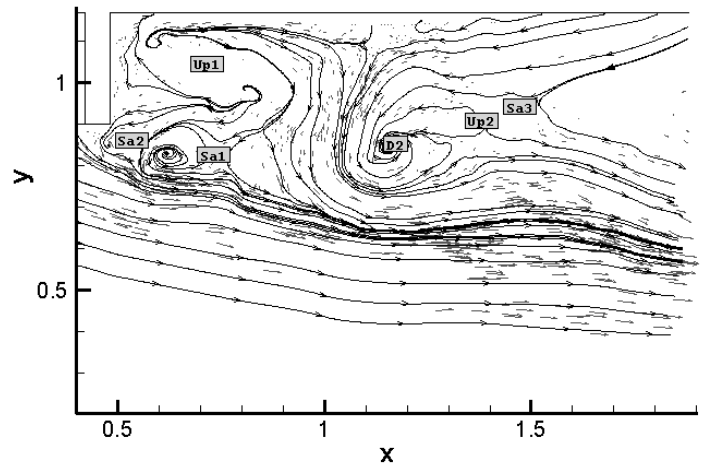

(b)
(The flow direction is left to right.)

(The horizontal coordinates $x$ and $y$ are given in meters.) 
and the groyne wake the particles had been deployed so that they could enter the test section near the groyne tip (from the left in Fig. 67) with an approximately optimum density, as discussed above. In Fig. 6a one can easily identify from bottom to top the fast main stream, the shear layer (with a freshly shed vortex) and a slow, but complex structure in the wake of the groyne. In Fig. 6p the immediate wake behind the groyne is blown up. In addition to the speed differences mentioned above, the unsteadiness of the flow can also be observed: during this short time interval several particle trajectories crossed each other, this would have been impossible in a steady flow.

What is even more conspicuous in Fig. 6, is the uneven distribution of the particles. Lagrangian particles drifted with the flow have a tendency to accumulate along the Lagrangian unstable manifolds of the hyperbolic points, at the same time they tend to evacuate the surrounding of the stable manifolds thereof. In addition to such hyperbolic structures, well-known in the theory of dynamical systems and conventional fluid dynamics, free surface flows provide another mechanism for attractive and repelling motion: upwelling and downwelling vortices. These features, not uncommon in fluvial environment, convey material to and from the surface, therefore the surface flow in general is not divergence free. Their behaviour can be best described mathematically as unstable and stable focus points, in the context of dynamical systems. An unstable (stable) focus can be considered as a combination of a source (sink) and a point vortex, and corresponds to an upwelling (downwelling) flow structure, respectively, and induces an outbound (inbound) spiralling motion on the surface. Since floaters, unlike water, have no supply within the water body and cannot leave the surface, they tend to depart quickly from upwelling regions, while downwellings attract them. In this manner some flow trajectories, possibly including the stable manifolds of Lagrangian hyperbolic points, can originate from unstable focus points, while others, like unstable manifolds, can lead into stable foci. In Fig. 7, we attempted to demonstrate this by reconstructing the instantaneous streamlines of the flow field at the beginning (in Fig. 7a) and at the end (in Fig. 7p) of the time period used in Fig. 6. The instantaneous flow fields were reconstructed by an interpolation scheme from the measured velocity of the individual particles. The saddle points, downwelling and upwelling foci are marked by 'Sa', 'D' and 'Up' in Fig. 7 respectively. There is an obvious displacement of these points between Figs. $7 \mathrm{a}$ and $7 \mathrm{p}$; this also reflects the fact that these structures are unsteady flow features, being in perpetual change. These kinds of structures and the corresponding buoy motions were observed during the field experiments in several cases. Thus, we can conclude that the laboratory experiments are convincingly reflecting the behaviour of the true river flow.

\section{Conclusions and discussion}

In order to detect chaotic advection, we have conducted field experiments in River Danube, Hungary. Lagrangian surface buoys with GPS receivers were released in a test section, which contained two groynes. A detailed analysis of the trajectories was carried out, focusing on the time evolution of the distance of the particle pairs. In spite of the small number of buoys, we found evidence on the chaotic features of particle dispersion in the wake of a groyne field. Based on the reconstructed buoyto-buoy distance time series, the analysis included the determination and comparison of the local Lyapunov exponents and the corresponding prediction time. High enough values of the local Lyapunov exponents for convincingly long enough times are considered reliable indicators of strongly dispersive hyperbolic motion. We have also identified occasionally buoy pairs, which were seemingly trapped in the same vortex. Such intermingling of hyperbolic and elliptic structures is characteristic to the complex mixing processes that are known to occur in groyne fields.

The experiments collected data in case of completely different hydraulic conditions. We have compared the spatial distribution of mixing strength in cases of emergent and submerged groynes at different water levels. If the groynes are dry, the mixing is governed by the wake field and the shear layer, and the durations of stretching periods are typically short. When the groynes are flooded, local turbulence acts as the source of dispersion, leading to a qualitatively different flow field and buoy tracks. The water depth covering the groynes proved to have a significant effect on the measured mixing strength. It is worth underlining, however, that the surface buoys sampled only the uppermost layer of the flow, offering a near-surface indicator on the mixing.

Laboratory experiments in a long, straight channel model were also performed to study these mechanisms in a controlled environment. A large number of floating tracer particles was followed by PTV technique. This analysis provides Lagrangian data, which are directly comparable to those of the field experiments. The high density of the particles made possible to identify moving saddle points, down- and upwelling vortices, detached shear layer vortices etc. in the surface flow. In fact, the high number of particles that are present in the test section at the same time would require the algorithmic identification and classification of the admissible exponential departures of floater pairs; this development is underway.

After these promising experiments, we plan to carry out further measurement campaigns in the same test section of the river. The forthcoming campaigns would target pre-designed flow conditions and specific locations to widen the range of information on the surface dynamics. These campaigns will hopefully involve more buoys and will be supplemented by parallel hydraulic monitoring and ADCP surveys. Notwithstanding, the amount of field information on the Lagrangian structures of fluvial surface motion will remain limited. In order to supplement our knowledge in this field we need to continue the small scale laboratory modelling. In addition, numerical experiments can serve as further sources of information: they can provide full depth and free surface velocity data. For a successful analy- 
sis, CFD tools with appropriate capabilities are necessary: three dimensional modelling ability, free surface handling, satisfactory boundary layer and turbulence models. For the latter, the application of LES seems to be necessary, since conventional parameterised turbulence models cannot predict deterministic Lagrangian trajectories: for such a purpose, the flow structures have to be resolved sufficiently. The development of such CFD tools is underway. The CFD models of the test section can be calibrated and validated against e.g. ADCP data as well as buoy trajectories. Successful numerical model could provide valuable information on mixing in similarly complex river geometries. Hyperbolic Lagrangian structures, like stable and unstable manifolds, and elliptic regions of the flow that hinder material transfer can be used to examine the mixing properties in different flow regimes.

\section{Acknowledgments}

This work is connected to the scientific program of the "Development of quality-oriented and harmonized $\mathrm{R}+\mathrm{D}+\mathrm{I}$ strategy and functional model at BME" project. This project is supported by the New Széchenyi Plan (Project ID: TáMOP-4.2.1/B09/1/KMR-2010-0002). Further support has been received from the Hungarian Scientific Research Fund (OTKA) under grant numbers NK72037 and K81621.

\section{References}

1 Engelhardt C, Krüger A, Sukhodolov A, Nicklisch A, A study of phytoplankton spatial distributions, flow structure and characteristics of mixing in a river reach with groynes, Journal of Plankton Research 26 (2004), no. 11, 1351-1366, DOI 10.1093/plankt/fbh125, available at http: //plankt.oxfordjournals.org/content/26/11/1351.abstract

2 Kurzke M, Weitbrecht V, Jirka G H, Laboratory concentration measurements for determination of mass exchange between groin fields and main stream, [1st] International Conference on Fluvial Hydraulics (Louvain-laNeuve, Belgium, 2002), River Flow 2002 (Bousmar D, Zech Y, eds.), A. A. Balkema Publishers, Lisse, The Netherlands, 2002, pp. 369-376.

3 Aref H, Stirring by chaotic advection, Journal of Fluid Mechanics 143 (1984), 1-21, DOI 10.1017/S0022112084001233, available at http:// journals.cambridge.org/abstract_S0022112084001233

4 van Mazijk A, Modelling the effects of groyne fields on the transport of dissolved matter within the Rhine Alarm-Model, Journal of Hydrology 264 (2002), no. 1-4, 213-229, DOI 10.1016/S0022-1694(02)00077-X, available at http://www.sciencedirect.com/science/article/pii/ S002216940200077X

5 Pattantyús-Ábrahám M, Tél T, Krámer T, Józsa J, Mixing properties of a shallow basin due to wind-induced chaotic flow, Advances in Water Resources 31 (2008), no. 3, 525-534, DOI 10.1016/j.advwatres.2007.11.00, available at http://www.sciencedirect.com/science/article/pii/ S0309170807001728

6 Sokoray-Varga B, Józsa J, Particle tracking velocimetry (PTV) and its application to analyse free surface flows in laboratory scale models, Periodica Polytechnica Civil Engineering 52 (2008), no. 2, 63-71, DOI 10.3311/pp.ci.2008-2.02, available at http://www.pp.bme.hu/ci/2008_ 2/ci2008_2_02.html

7 Tél T, Gruiz M, Chaotic dynamics, Cambridge University Press, 2006, available at http://www.cambridge.org/gb/knowledge/isbn/ item1112815/
8 Uijttewaal W S J, Lehmann D, van Mazijk A, Exchange Processes between a River and Its Groyne Fields: Model Experiments, Journal of Hydraulic Engineering 127 (2001), no. 11, 928-936, DOI 10.1061/(ASCE)0733-9429(2001)127:11(928).

9 Uijttewaal W S J, Effects of Groyne Layout on the Flow in Groyne Fields: Laboratory Experiments, Journal of Hydraulic Engineering 131 (2005), no. 9, 782-791, DOI 10.1061/(ASCE)0733-9429(2005)131:9(782).

10 Weitbrecht V, Kühn G, Jirka G H, Large scale PIV-measurements at the surface of shallow water flows, Flow Measurement and Instrumentation 13 (2002), no. 5-6, 237-245, DOI 10.1016/S0955-5986(02)00059-6.

11 Weitbrecht $\mathbf{V}$, Influence of dead-water zones on the dispersive masstransport in rivers, Universitätsverlag Karlsruhe, 2004, available at http: //digbib.ubka.uni-karlsruhe.de/volltexte/1242004/

\section{Appendix}

In this Appendix we list all those finite-time intervals during which a definite positive Lyapunov exponent can be identified in the distance time series of a buoy pair.

Tab. 2. $1^{\text {st }}$ measurement campaign, 2009-11-04

\begin{tabular}{ccccccc}
\hline $\begin{array}{c}\text { Campaign/ } \\
\text { release }\end{array}$ & $\begin{array}{c}\text { Start } \\
{[\mathbf{s}]}\end{array}$ & $\begin{array}{c}\text { End } \\
\text { [s] }\end{array}$ & $\begin{array}{c}\text { Duration } \\
{[\mathbf{s}]}\end{array}$ & Buoys & $\begin{array}{c}\text { Lyapunov } \\
\text { exponent }\left[\mathbf{s}^{-1} \text { ] }\right.\end{array}$ & $\mathbf{R}^{2}$ \\
\hline $1 / 1$ & 5 & 34 & 29 & $07-08$ & 0.044 & 0.96 \\
\hline $1 / 1$ & 51 & 105 & 54 & $06-07$ & 0.021 & 0.97 \\
\hline $1 / 1$ & 96 & 236 & 140 & $06-08$ & 0.015 & 0.99 \\
\hline $1 / 3$ & 58 & 110 & 52 & $06-08$ & 0.025 & 0.94 \\
\hline $1 / 6$ & 88 & 131 & 43 & $07-08$ & 0.031 & 0.98 \\
\hline
\end{tabular}

Tab. 3. $1^{\text {st }}$ measurement campaign, 2009-11-05

\begin{tabular}{ccccccc}
\hline $\begin{array}{c}\text { Campaign/ } \\
\text { release }\end{array}$ & $\begin{array}{c}\text { Start } \\
{[\mathbf{s}]}\end{array}$ & $\begin{array}{c}\text { End } \\
\text { [s] }\end{array}$ & $\begin{array}{c}\text { Duration } \\
\text { [s] }\end{array}$ & Buoys & $\begin{array}{c}\text { Lyapunov } \\
\text { exponent [s }{ }^{-1} \text { ] }\end{array}$ & $\mathbf{R}^{2}$ \\
\hline $1 / 7$ & 100 & 217 & 117 & $07-08$ & 0.014 & 0.99 \\
\hline $1 / 7$ & 164 & 324 & 160 & $06-08$ & 0.012 & 0.99 \\
\hline $1 / 8$ & 0 & 60 & 60 & $06-07$ & 0.047 & 0.96 \\
\hline $1 / 8$ & 135 & 455 & 320 & $07-08$ & 0.007 & 0.98 \\
\hline $1 / 8$ & 146 & 438 & 292 & $06-07$ & 0.004 & 0.99 \\
\hline
\end{tabular}


Tab. 4. $2^{\text {nd }}$ measurement campaign, 2010-08-12

\begin{tabular}{|c|c|c|c|c|c|c|}
\hline $\begin{array}{c}\text { Campaign/ } \\
\text { release }\end{array}$ & $\begin{array}{c}\text { Start } \\
\text { [s] }\end{array}$ & $\begin{array}{l}\text { End } \\
{[\mathbf{s}]}\end{array}$ & $\begin{array}{l}\text { Duration } \\
\text { [s] }\end{array}$ & Buoys & $\begin{array}{c}\text { Lyapunov } \\
\text { exponent }\left[\mathbf{s}^{-1}\right]\end{array}$ & $\mathbf{R}^{2}$ \\
\hline $2 / 01$ & 60 & 290 & 230 & 03-06 & 0.006 & 0.94 \\
\hline $2 / 01$ & 60 & 340 & 280 & 02-08 & 0.006 & 0.99 \\
\hline $2 / 01$ & 60 & 340 & 280 & 06-08 & 0.008 & 0.98 \\
\hline $2 / 01$ & 370 & 718 & 348 & $02-06$ & 0.004 & 0.98 \\
\hline $2 / 01$ & 481 & 718 & 237 & 03-06 & 0.005 & 0.98 \\
\hline $2 / 02$ & 120 & 214 & 94 & $02-08$ & 0.016 & 0.95 \\
\hline $2 / 02$ & 125 & 421 & 296 & $02-06$ & 0.004 & 0.98 \\
\hline $2 / 02$ & 125 & 421 & 296 & $02-03$ & 0.004 & 0.96 \\
\hline $2 / 03$ & 30 & 66 & 36 & 06-08 & 0.060 & 0.98 \\
\hline $2 / 03$ & 30 & 66 & 36 & $02-06$ & 0.060 & 0.996 \\
\hline $2 / 03$ & 80 & 107 & 27 & $02-03$ & 0.060 & 0.99 \\
\hline $2 / 04$ & 283 & 388 & 105 & 03-06 & 0.020 & 0.94 \\
\hline $2 / 04$ & 300 & 430 & 130 & $02-03$ & 0.010 & 0.96 \\
\hline 2/07 & 33 & 140 & 107 & 03-09 & 0.017 & 0.92 \\
\hline $2 / 07$ & 42 & 140 & 98 & 06-09 & 0.030 & 0.98 \\
\hline $2 / 07$ & 156 & 840 & 684 & 06-07 & 0.004 & 0.99 \\
\hline $2 / 07$ & 372 & 840 & 468 & $02-07$ & 0.004 & 0.99 \\
\hline $2 / 07$ & 450 & 588 & 138 & $02-03$ & 0.009 & 0.99 \\
\hline $2 / 09$ & 300 & 561 & 261 & 03-07 & 0.007 & 0.94 \\
\hline $2 / 09$ & 330 & 550 & 220 & 07-09 & 0.009 & 0.94 \\
\hline
\end{tabular}

Tab. 5. $2^{\text {nd }}$ measurement campaign, 2010-08-13

\begin{tabular}{ccccccc}
\hline $\begin{array}{c}\text { Campaign/ } \\
\text { release }\end{array}$ & $\begin{array}{c}\text { Start } \\
{[\mathbf{s}]}\end{array}$ & $\begin{array}{c}\text { End } \\
{[\mathbf{s}]}\end{array}$ & $\begin{array}{c}\text { Duration } \\
{[\mathbf{s}]}\end{array}$ & Buoys & $\begin{array}{c}\text { Lyapunov } \\
\text { exponent }\left[\mathbf{s}^{-1}\right]\end{array}$ & $\mathbf{R}^{2}$ \\
\hline $2 / 10$ & 0 & 250 & 250 & $03-06$ & 0.010 & 0.96 \\
\hline $2 / 10$ & 0 & 250 & 250 & $06-09$ & 0.010 & 0.98 \\
\hline $2 / 11$ & 0 & 225 & 225 & $06-07$ & 0.010 & 0.95 \\
\hline $2 / 11$ & 105 & 276 & 171 & $02-07$ & 0.016 & 0.95 \\
\hline $2 / 11$ & 426 & 460 & 34 & $07-09$ & 0.050 & 0.95 \\
\hline $2 / 11$ & 641 & 751 & 110 & $07-09$ & 0.012 & 0.99 \\
\hline $2 / 12$ & 10 & 425 & 415 & $02-06$ & 0.005 & 0.95 \\
\hline $2 / 12$ & 22 & 280 & 258 & $03-06$ & 0.007 & 0.97 \\
\hline $2 / 12$ & 190 & 480 & 290 & $02-03$ & 0.007 & 0.94 \\
\hline $2 / 12$ & 310 & 1081 & 771 & $07-09$ & 0.003 & 0.98 \\
\hline $2 / 12$ & 480 & 840 & 360 & $03-07$ & 0.004 & 0.99 \\
\hline $2 / 13$ & 499 & 620 & 121 & $03-06$ & 0.010 & 0.98 \\
\hline $2 / 13$ & 586 & 797 & 211 & $07-09$ & 0.006 & 0.99 \\
\hline $2 / 14$ & 106 & 173 & 67 & $03-07$ & 0.040 & 0.96 \\
\hline $2 / 14$ & 390 & 540 & 150 & $07-09$ & 0.010 & 0.95 \\
\hline $2 / 14$ & 480 & 610 & 130 & $02-03$ & 0.009 & 0.96 \\
\hline $2 / 14$ & 680 & 1160 & 480 & $06-07$ & 0.003 & 0.99 \\
\hline $2 / 14$ & 760 & 1220 & 460 & $03-07$ & 0.004 & 0.96 \\
\hline & & & & & &
\end{tabular}

